Mens

Revue d'histoire intellectuelle et culturelle

mens

\title{
Spectacles de variétés et arrivée de la télévision à Saint-Hyacinthe, 1950-1968
}

\section{Guy Gaudreau}

Volume 18, numéro 1, automne 2017

URI : https://id.erudit.org/iderudit/1062933ar

DOI : https://doi.org/10.7202/1062933ar

Aller au sommaire du numéro

Éditeur(s)

Centre de recherche en civilisation canadienne-française

ISSN

1492-8647 (imprimé)

1927-9299 (numérique)

Découvrir la revue

Citer cet article

Gaudreau, G. (2017). Spectacles de variétés et arrivée de la télévision à

Saint-Hyacinthe, 1950-1968. Mens, 18(1), 83-110.

https://doi.org/10.7202/1062933ar

\section{Résumé de l'article}

Basé sur un dépouillement des publicités des deux hebdomadaires de Saint-Hyacinthe, Le Clairon et Le Courrier de Saint-Hyacinthe, l'article aborde l'évolution, entre 1950 et 1968, des spectacles de variétés maskoutains, autant ceux qui se déroulent dans les hôtels sous la forme de cabaret que ceux présentés dans les salles de cinéma. Cet examen parallèle permet de bien cerner l'éventail et la nature de ces spectacles. Si les rares travaux s'intéressant à ce sujet se sont surtout penchés sur la situation des spectacles de variétés à Montréal, nos résultats montrent que ceux-ci n’ont pas connu la même évolution que ceux qui étaient présentés à l'extérieur de la métropole et que la télévision, au début des années 1960 - avec le lancement de Télé-Métropole en 1961 et un changement d'orientation à Radio-Canada -, a joué un rôle marquant dans leur déclin, tant dans les cabarets que dans les salles de cinéma. 


\title{
Spectacles de variétés et arrivée de la télévision à Saint-Hyacinthe, 1950-1968
}

\author{
Guy Gaudreau \\ Université Laurentienne
}

\section{Résumé}

Basé sur un dépouillement des publicités des deux hebdomadaires de Saint-Hyacinthe, Le Clairon et Le Courrier de Saint-Hyacinthe, l'article aborde l'évolution, entre 1950 et 1968, des spectacles de variétés maskoutains, autant ceux qui se déroulent dans les hôtels sous la forme de cabaret que ceux présentés dans les salles de cinéma. Cet examen parallèle permet de bien cerner l'éventail et la nature de ces spectacles. Si les rares travaux s'intéressant à ce sujet se sont surtout penchés sur la situation des spectacles de variétés à Montréal, nos résultats montrent que ceux-ci n'ont pas connu la même évolution que ceux qui étaient présentés à l'extérieur de la métropole et que la télévision, au début des années 1960 - avec le lancement de Télé-Métropole en 1961 et un changement d'orientation à RadioCanada -, a joué un rôle marquant dans leur déclin, tant dans les cabarets que dans les salles de cinéma.

Parmi les sujets encore peu abordés en histoire culturelle au Québec, il y a assurément celui des spectacles de variétés ${ }^{1}$. Que des

\footnotetext{
${ }^{1}$ Le Traité de la culture, par exemple, ne compte aucun article sur le sujet bien qu'il consacre quatre chapitres aux arts de la scène, soit les institutions (chap. 39), 
spectacles aussi courus aient fait l'objet de si peu de recherches pourra encore étonner. Divertissements populaires, les numéros de variétés peuvent être associés à deux genres d'art de la scène qui ont été grandement influencés par la pratique étatsunienne : le théâtre burlesque $^{2}$, qui remonte au XIX ${ }^{\mathrm{e}}$ siècle, et le cabaret, qui connaît son apogée à Montréal au cours des années $1940^{3}$.

Ce genre de spectacles offre un programme varié $e^{4}$ qui comprend des sketches, de la danse, du chant et, selon les besoins, des numéros de cirque et de magie. Les spectacles de variétés sont habituellement présentés sur la scène d'une salle de cinéma et dans les hôtels où ils adoptent la formule bien connue du cabaret alors que, dans un cinéma, ils peuvent relever du théâtre burlesque ou du vaudeville 5 . Pour éviter toute confusion, nous nous en tiendrons au concept de spectacle de variétés en examinant parallèlement les spectacles qui sont produits dans les hôtels et dans les cinémas, d'autant plus que, comme on le verra, les artistes, à une certaine époque, passent fréquemment d'un lieu à l'autre.

le théâtre (chap. 40), la danse (chap. 41) et le cirque (chap. 42). Denise Lemieux (dir.), Traité de la culture, Québec, Les Éditions de l'IQRC/Les Presses de l'Université Laval, 2002, p. 739-798.

2 Chantal Hébert a consacré son mémoire de maîtrise et sa thèse de doctorat à ce sujet. Voir Le burlesque au Québec : un divertissement populaire, Montréal, Hurtubise HMH, 1981 et Le burlesque québécois et américain : textes inédits, Québec, Les Presses de l'Université Laval, 1989. Elle a également publié avec Gérard Thibault, Chez Gérard : la petite scène des grandes vedettes, 1938-1978, Sainte-Foy, Les Éditions spectaculaires, 1988. On pourra lire aussi l'article plus récent de Sylvain de Champlain, "Le burlesque au Québec : Pic-Pic, Pizzy-Wizzy, Swifty... », À rayons ouverts - Chroniques de Bibliothèque et Archives nationales du Québec, no 92 (printemps-été 2013), p. 13-15.

3 William Weintraub, City Unique: Montreal Days and Nights in the 1940s and '50s, Toronto, Robin Brass Studio, 2004 [1996]; Nancy Marrelli, Stepping Out: The Golden Age of Montreal Night Clubs, Montréal, Véhicule Press, 2004.

${ }^{4}$ Selon Le Dictionnaire historique de la langue française : "En 1790 est créé le Théâtre des Variétés de $\mathrm{M}^{\mathrm{me}}$ Montansier, dont le répertoire "varié" était surtout composé de vaudevilles; par extension, le mot désigne (1913) l'ensemble des activités de spectacle concernant un type de chansons, de spectacles comiques, etc. destiné à un large public ", p. 2216.

5 Sur cette question, voir Hébert, Le burlesque au Québec, p. 7-9. 
Bien que les connaissances sur le sujet demeurent modestes, on peut affirmer qu'elles sont de deux ordres : d'une part, elles décrivent les spectacles qui se déroulent à Montréal $^{6}$ et, d'autre part, elles proviennent des récits des principaux artisans de la scène. Ainsi, pour affirmer que le « théâtre burlesque » décline au cours des années 1950, on évoque la vente du Théâtre National de Rose "La Poune " Ouellette, haut lieu du théâtre burlesque montréalais, en $1953^{7}$ et celle, trois ans plus tard, du théâtre Radio-Cité, propriété de Jean Grimaldi $^{8}$. D'autres témoignages ${ }^{9}$ vont dans le même sens, comme ceux de Jacques Normand ${ }^{10}$, célèbre animateur des cabarets de Montréal, de Juliette Petrie ${ }^{11}$, de Jean Rafa ${ }^{12}$, de Denyse Émond ${ }^{13}$ (Ti-Mousse) et celui, plus récent, de Denise Filiatrault ${ }^{14}$.

Mis à part les travaux de Hébert, peu d'études se sont intéressées au sujet. Si l'on a pu dresser un portrait d'ensemble des spectacles de la Main ${ }^{15}$, les recherches sur les cabarets sont rares. Robert Aird avait

${ }^{6}$ Ce « montréalocentrisme » dans le traitement de la culture est aussi présent dans le dossier de la revue Mens, paru à l'hiver 2017 et consacré à la «Vie musicale amateur, populaire et américaine à Montréal, 1918-1958». On trouvera toutefois plusieurs références utiles dans l'étude sur la musique montréalaise signée par la directrice du dossier. Voir Sandria P. Bouliane, "Vie musicale amateur, populaire et américaine à Montréal, 1918-1958 ", Mens : revue d'histoire intellectuelle et culturelle, vol. XVI, ${ }^{\circ} 1$ (automne 2015), p. 7-14.

7 Rose Ouellette, «La Poune », Montréal, Éditions Québécor, 1983, p. 71-72.

8 Autrefois le théâtre Gayeté, le Radio-Cité deviendra La Comédie-Canadienne (Jean Grimaldi présente, propos recueillis par Jacques Cimon et Philippe Laframboise, Montréal, René Ferron éditeur, 1973, p. 73-77).

9 En plus de l'ouvrage cité à la note précédente, on pourra également lire : "Jean Grimaldi, le "papa des artistes" ", propos recueillis par Yves Beauregard, Cap-auxDiamants, la revue d'histoire du Québec, no 35 (automne 1993), p. 21-25.

${ }^{10}$ Jacques Normand, Les nuits de Montréal, Montréal, Les Éditions La Presse, 1974.

11 Juliette Petrie, Quand on revoit tout ça!, propos recueillis par Jean Leclerc, Montréal, Éditions J. Petrie, 1977.

12 Pierre Day, Jean Rafa, de Paris à Montréal, Louiseville, Les Éditions Logiques, 1997.

13 David Lavallée, Denyse Émond Ti-Mousse, Chicoutimi, Éditions JCL, 2009.

14 Denise Filiatrault et Danièle Lorain, Quand tu es née pour un petit pain, Montréal, Libre Expression, 2017.

15 Voir André-G. Bourassa et Jean-Marc Larrue, Les nuits de la "Main » : cent ans de spectacles sur le boulevard Saint-Laurent (1891-1991), Montréal, VLB éditeur, 1993. 
certes effleuré le sujet dans son étude sur l'évolution du monologue ${ }^{16}$, mais il a fallu attendre Luc Bellemare pour qu'une recherche soit consacrée spécifiquement à un cabaret montréalais du tournant des années $1930^{17}$.

Examinés sous le prisme de la question identitaire et souvent de la construction de la culture montréalaise, ces études et ces récits ont laissé peu de place aux régions, réduites généralement à des lieux de tournée. L'image qui s'en dégage relève presque du folklore ou du mythe : pour assurer leur gagne-pain, les artistes d'une troupe partent en tournée dans des conditions difficiles, parcourant parfois de longues distances, en se jouant des tours pendables et en se contentant de chambres d'hôtel qui ne payent pas de mine. Pourtant, l'étude récente de Catherine Lefrançois sur les spectacles de country-western montre bien le rôle des régions, qui ont longtemps soutenu cette forme de musique populaire ${ }^{18}$. Quelle est donc la contribution des régions à la pratique et à l'évolution des spectacles de variétés? Voilà une des questions posées ici.

Il nous a semblé que l'examen de ces spectacles gagnerait à être circonscrit à une seule ville afin de les inscrire dans une réalité concrète, qui permettrait d'aller plus loin que les seuls témoignages des artisans. D'où le choix de Saint-Hyacinthe. Considérant le peu d'attention accordée à ces spectacles dans l'historiographie maskoutaine ${ }^{19}$,

16 Robert Aird, "De Coutlée au stand up comique : l'évolution du monologue québécois, de 1900 à nos jours ", GLOBE, vol. 11, nº 2 (2008), p. 23-41.

17 Luc Bellemare, "De Montmartre à Broadway : le cabaret Au matou botté de Montréal (1929-1932) ", Mens : revue d'histoire intellectuelle et culturelle, vol. XVI, $\mathrm{n}^{\circ} 1$ (automne 2015), p. 63-105.

18 Voir Catherine Lefrançois, "À l'assaut de Montréal : les chanteurs country-western dans les studios de la métropole, 1942-1958", Mens : revue d'histoire intellectuelle et culturelle, vol. XVI, n 1 (automne 2015), p. 107-138.

19 En fait, on peut parler de silence complet sur le sujet, comme on peut le constater dans l'ouvrage de synthèse de Mario Filion et al., Histoire du Richelieu-YamaskaRive-Sud, Québec, Les Éditions de l'IQRC/Les Presses de l'Université Laval, 2001, coll. "Les régions du Québec " et dans celui de la Société d'histoire régionale de Saint-Hyacinthe, Saint-Hyacinthe, 1748-1998, Sillery, Éditions du Septentrion, 1998. 
l'exercice valait d'autant plus le coup. Située à une petite heure en voiture de Montréal, cette ville est susceptible d'accueillir les tournées de spectacles de variétés produits en métropole, tout en favorisant peut-être une offre de services culturels propres à la région. Ce choix permettra aussi de voir concrètement comment se sont déployés ces spectacles, quelles ont été leurs différentes formes, quels artistes y ont été invités et quels sont ceux qui y sont venus le plus souvent. La ville de Saint-Hyacinthe jouit d'un autre avantage puisqu'elle est dotée de deux hebdomadaires rivaux que nous connaissons bien ${ }^{20}$ et dont le dépouillement parallèle des pages culturelles a permis de bonifier la cueillette d'informations. Loin de nous l'idée d'analyser le discours ou encore le travail des publicitaires ${ }^{21}$. Il s'agira plutôt de cerner d'abord la nature des spectacles de variétés.

Au sujet de leur évolution, retenons que plusieurs artisans se sont plaints de l'arrivée de la télévision et de ses conséquences sur les arts de la scène. Ainsi, dès 1973, Jean Grimaldi affirmait : "Tout allait pour le mieux quand la télévision m'enleva tous mes chanteurs et mes comédiens à grands coups de cachets. Tous ces artistes [...] entrevirent soudainement le veau d'or, car ils touchaient plus en une seule émission que pour un mois d'engagement au théâtre ${ }^{22}$. » Nous centrons donc

20 Pour en savoir un peu plus sur ces deux hebdomadaires, voir Ronald Martel, Histoire de la presse hebdomadaire au Québec: Estrie-Montérégie, Montréal, Hebdos Québec, 2009, p. 72-85; Guy Gaudreau et Micheline Tremblay, «Technique de mise en pages, crise économique et journalisme d'opinion : Le Courrier de SaintHyacinthe de 1920 à 1938 ", Scientia Canadensis: Canadian Journal of the History of Science, Technology and Medicine = Revue canadienne d'histoire des sciences, des techniques et de la médecine, vol. 36, $\mathrm{n}^{\circ} 2$ (2013), p. 37-62.

${ }^{21}$ Comme nous le verrons, selon les données recueillies, les publicités ne sont pas seulement diffusées, mais également produites par ces journaux puisqu'elles sont montées dans un encadré fait maison. Il ne s'agit donc pas à proprement parler d'annonces publicitaires, si l'on entend par là le produit d'un graphiste et d'une agence publicitaire embauchés pour l'occasion; nous préférons donc parler d'encadrés publicitaires. Sur l'utilisation des publicités par les historiens, on pourra lire Roland Marchand, Advertising the American Dream: Making Way for Modernity, 1920-1940, Berkeley, University of California Press, 1985.

22 Jean Grimaldi présente, p. 74-75. Dans son autobiographie, Denise Filiatrault tiendra sensiblement le même discours, voir Filiatrault et Lorain, Quand tu es née pour un petit pain, p. 88 et 100. 
notre démarche sur l'hypothèse que l'arrivée de la télévision a eu des incidences importantes sur les arts de la scène. Il convient donc d'amorcer la réflexion quelques années avant l'arrivée du petit écran; c'est pourquoi nous avons choisi, un peu arbitrairement, l'année 1950 afin de définir l'offre de spectacles de variétés à cette époque.

\section{Des spectacles épisodiques : 1950-1953}

En 1950, la ville compte, depuis quelques décennies, deux salles de cinéma. Le Corona, construit par l'homme d'affaires et député libéral T.-D. Bouchard, ouvre ses portes en 1916 et le cinéma Maska, en $1937^{23}$. En fait, l'un et l'autre accueillent des spectacles que l'on peut qualifier de variétés depuis un bon moment.

Arrêtons-nous, à titre d'exemple, sur une année prise au hasard : 1938. "Dimanche seulement, le 30 octobre, il y aura des représentations de vaudeville au Maska dans l'après-midi et la soirée. Ces représentations de vaudeville le dimanche sont fort populaires au théâtre de la rue Sainte-Catherine et la direction nous promet pour dimanche prochain des numéros de tout premier ordre ${ }^{24}$. "Parfois la publicité ne mentionne pas le nom des artistes, il est alors difficile de savoir quel type de spectacles est offert. En revanche, quand les artistes sont nommés, on peut aisément déterminer quels sont les numéros de variétés qui seront présentés (voir la figure 1). On peut aussi noter que ces représentations ont lieu de manière très irrégulière. Et cela semble être aussi le cas au cours des années suivantes.

Faisons maintenant le point sur la situation en 1950. L'économie de Saint-Hyacinthe est incontestablement prospère. Ainsi, entre 1950 et 1954, on double la capacité d'accueil lorsque le Centre NotreDame, avec son auditorium, ouvre ses portes le 25 décembre 1950 et que le Corona ajoute 500 sièges en mai 1952. Puis, en janvier 1954, c'est l'inauguration d'un nouveau cinéma, Le Paris, qui peut accueillir 920 personnes. On est encore loin de la baisse de fréquen-

\footnotetext{
${ }^{23}$ Société d'histoire régionale de Saint-Hyacinthe, Saint-Hyacinthe, 1748-1998, p. 364.

${ }^{24}$ Le Clairon, 28 octobre 1938, p. 8.
} 
tation que vont encaisser les salles de cinéma en raison de la popularité du petit écran qui, rappelons-le, diffuse pour la première fois le 6 septembre 1952. La hausse du niveau de vie de la classe ouvrière de Saint-Hyacinthe après la guerre favorise une plus grande consommation, notamment, de produits culturels.
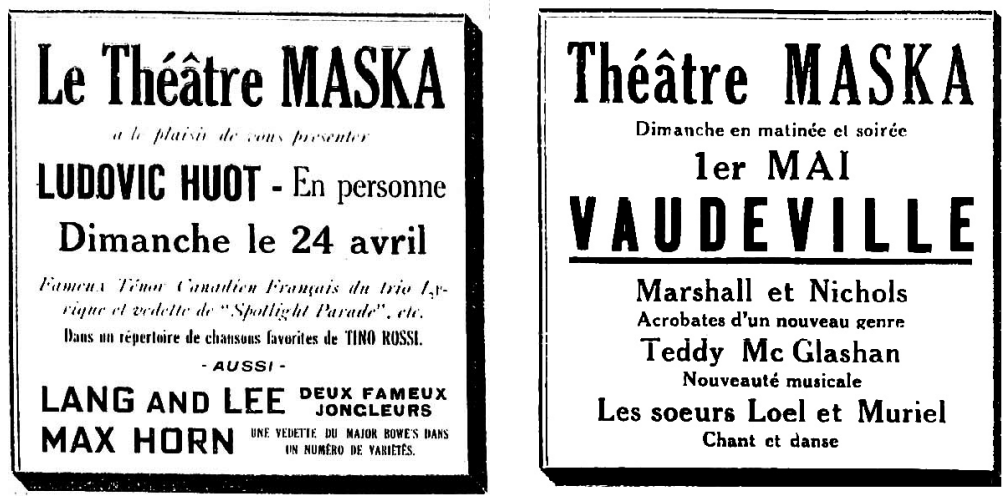

Figure 1 - Deux encadrés publicitaires du printemps 1938.

Sources : Le Clairon, 22 avril 1938, p. 2; Le Clairon, 29 avril 1938, p. 3.

Le dépouillement systématique du Clairon et du Courrier de Saint-Hyacinthe pour l'année 1950 indique encore une présence occasionnelle des variétés. Comme elle le fait depuis plusieurs années, la troupe de Ti-Pit Raccourci vient dérider les Maskoutains le 22 avril. Cette fois, l'activité a lieu le samedi au cinéma Maska ${ }^{25}$. On accueille également ce genre de spectacles dans le cadre de deux événements. Le premier est l'exposition régionale agricole qui se déroule du 8 au 10 août et qui offre une "Grande revue "Variétés 1950" " avec, en vedette, Muriel Millard et de nombreux numéros de cirque, comme celui du capitaine Tiebor et de ses phoques savants ${ }^{26}$. Le second événement est une parade de mode de la maison Bernard, qui emprunte la scène du cinéma Maska le mercredi 13 septembre. Au

25 L'année suivante, c'est au Corona qu'il donne son spectacle. Voir Le Clairon, 30 novembre 1951, p. 14.

${ }^{26}$ Le Clairon, 4 août 1950, p. 14. 
sujet de cette dernière, on écrit : «Une heure de gaieté avec Jacques Normand, Monique Leyrac (chanteuse), Gilles Pellerin (comédien), Billy Munro (pianiste fantôme) aussi Larry Aubin (et son orchestre) ${ }^{27}$ ".

Si l'on en croit les publicités des deux hebdomadaires, il n'y aurait pas encore, au début des années 1950, de spectacles de variétés dans les hôtels maskoutains. Quand quelques artistes, qui brûlent les planches des cabarets de Montréal, viennent en ville, leur performance est toujours goûtée dans une salle de cinéma. Ainsi, le mardi 14 novembre, le Maska reçoit la tournée d'adieu des «Vedettes en balade » avec Nelly Mathot, Clairette, Jean Rafa, Pierre Roche et le "prince des annonceurs ", Saint-Georges Côtée ${ }^{28}$.

Pourtant, on s'explique mal leur absence dans les hôtels maskoutains alors que la formule du cabaret bat son plein à Montréal. Peut-on y voir une plus grande flexibilité des salles du $7^{\mathrm{e}}$ art, qui doivent constamment changer leur programmation, sans compter qu'elles ont la capacité d'accueillir un large public, garantie d'une meilleure rentabilité?

Après la vente de son théâtre, en 1953, abandonnant ainsi sa propre salle, La Poune part en tournée avec sa troupe et s'arrête à deux reprises au cinéma Corona, les vendredis 24 juillet et 4 septembre 1953 (voir la figure 2). Le premier spectacle a du succès si l'on en croit le communiqué émis à l'occasion de son retour : "À la demande générale $[\ldots]$ dans un spectacle entièrement nouveau ${ }^{29} »$. Lannonce ne précise pas si des films, qui font traditionnellement partie du théâtre burlesque $^{30}$, sont au programme du spectacle d'une durée de trois heures.

${ }^{27}$ Le Clairon, 8 septembre 1950, p. 5.

${ }^{28}$ Le Clairon, 10 novembre 1950, p. 5. Cet événement n'est pas sans rappeler la présence de Jacques Normand deux mois plus tôt au même endroit.

29 Le Clairon, 4 septembre 1953, p. 8.

30 Pour une belle description du déroulement d'un spectacle burlesque, voir Ouellette, "La Poune", p. 67-71. Voici ce qu'elle dit du spectacle après le visionnement des films : «D'abord l'ouverture musicale, avec tous les membres de la troupe. Puis venait un petit drame - en fait le plus souvent un mélodrame - qui précédait une série de petits sketches d'une dizaine de minutes chacun. Suivaient les "attractions" : acrobates, danseurs, chanteurs, magiciens ou hypnotiseurs. Enfin, le clou du spectacle, ce qu'on appelait la grande comédie. » (p. 70) 
Le 23 octobre, c'est au tour de la troupe d'Oswald de divertir pour la première fois les spectateurs maskoutains avec "En avant marche ", qui comprend bouffons, acrobates, danseuses, chants, musique et comédies $^{31}$. Si les spectacles de variétés offerts dans les cinémas agonisent à Montréal, ils sont encore bien populaires en région.

TEL. 4-9404 SAINT-HYACINTHE, QUE.

\section{THE A T RE}

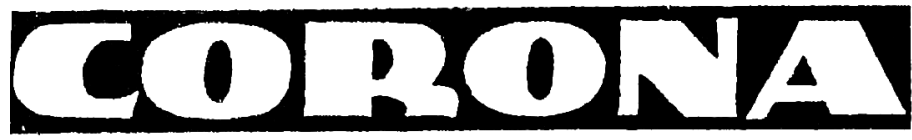

LE FOYER DU FILM PARLANT FRANÇAIS

Vendredi soir, 24 juillet, à 8.30 hres "LA POUNE ET SA TROUPE" dans un spectacle de 3 heures.

CHANT - DANSE - MUSIQUE - COMEDIE

La fameuse POUNE du National, surnommée "La Bombe Atomique du Rire". Un Spectacle que vous n'oublierez jamais Admission: $\$ 1.00$ of $\$ 0 . \overline{7} 5$ - Taxes incluses. Billels actuellement en vente au Théatre.

Figure 2 - « La Poune et sa troupe », Le Clairon, 17 juillet 1953, p. 7.

De nouveau sur la scène du Corona le 4 juin 1954, La Poune boude les Maskoutains en 1955. L'année suivante, elle se joint à la troupe de Jean Grimaldi qui, en difficulté financière, vient à son tour

31 Le Courrier de Saint-Hyacinthe, 16 octobre 1953, p. 21. 
de vendre son théâtre pour partir en tournée. Leur spectacle prend l'affiche au Paris le lundi 31 décembre 1956. Les artistes qui y participent nous donnent une idée de la diversité des numéros présentés, qui, de toute évidence, n'incluent pas la projection d'un film. En effet, on peut y voir : "La grande comédienne La Poune, le comédien de l'heure Bazou, Maurice Gauvin, l'Oncle Albert de "14, rue des Galais", Paolo Noël, la voix de Tino Rossi, Carole Mercure, notre Édith Piaf, Roger Miron et ses Laurentiens, Liliane Dawson, la bombe atomique de la chanson, Les Clover Boys, les rois du Rock and Roll et du Cha-Cha-Cha, Jean Robi, le champion du tap-dance, la charmante et talentueuse Lucille Serval, le fantaisiste Paul Thériault, et Nick Pensato et son orchestre ${ }^{32}$."

Venue quelques semaines plus tôt (le 4 décembre), la troupe de Jean Grimaldi ${ }^{33}$ revient à douze reprises au Paris, entre le 19 janvier et le 11 mai $1957^{34}$ et toujours sans La Poune. Plus souvent qu'autrement, les publicités n'ont pas d'intitulé et on y évoque à quatre reprises ce qui est alors un genre musical nouveau, le rock and roll. Autre détail : la mention de deux films en complément de programme y figure la moitié du temps.

Le 11 mai, on annonce : "vos artistes préférés que vous voyez à la télévision »; et pour le 25 janvier, les sensationnels Avalons, participants au Ed Sullivan Show. La télévision, qu’elle soit étatsunienne,

32 "Le Grand Gala de la veille du Jour de l'An », Le Courrier de Saint-Hyacinthe, le 28 décembre 1956 , p. 5.

33 Jean Grimaldi vient à Saint-Hyacinthe pour la première fois au cours des années 1950, précisément le 22 avril 1955, alors qu'il présente un spectacle mettant en vedette Rina Ketty, entourée de Manda et des artistes de son théâtre RadioCité (Le Courrier de Saint-Hyacinthe, le 7 avril 1955, p. 7). Précisons qu'un dépouillement partiel du fonds d'archives Jean-Grimaldi ne nous a rien appris sur ses tournées maskoutaines, l'essentiel de la maigre correspondance relative aux tournées (MSS433, 53, SS1, D11) et aux publicités s'y rattachant (MSS433, 53, SS1, D15) porte sur Montréal ou encore se compose de lettres reçues à partir des années 1960.

${ }^{34}$ Soit les 19 et 26 janvier, les 2, 9, 16 et 23 février, les 2, 9, 16, 23 mars, le 5 avril et le 11 mai 1957. 
radio-canadienne anglaise ou française ${ }^{35}$, ne peut plus être ignorée. Les visites fréquentes de la troupe de Jean Grimaldi reflètent le grand intérêt du public maskoutain pour ces arts de la scène. Quant à la nature des spectacles offerts, ils paraissent cette année-là de plus en plus musicaux, peut-être moins comiques et, surtout, de moins en moins variés. Et compte tenu que les films sont projetés de manière irrégulière, leur présence ne paraît pas en être une composante essentielle.

\section{Des spectacles de variétés à l'hôtel}

Tout nous porte à croire que les hôtels et les salles de réception de Saint-Hyacinthe, qui offriront plus tard ces spectacles, sont encore, au début des années 1950, des lieux fréquentés pour la qualité des repas et la salle de danse. Une page publicitaire du Clairon maskoutain $^{36}$ du 18 octobre 1954 est, à cet effet, fort éclairante (voir la figure 3).

Quelques rares photographies nous font voir, en 1954, un orchestre composé de huit musiciens dirigés par Marcel Théberge au grill du Grand Hôtel ${ }^{37}$, tandis que Ray Laliberté en dirige un autre au grill dansant de l'Hôtel Ottawa ${ }^{38}$. Une artiste invitée vient chanter les vendredis et les samedis, mais il n'est pas encore question de variétés, du moins de manière régulière, car l'Hôtel Ottawa organise seulement deux soirées de variétés en 1954 : le 27 mars pour la micarême et le 27 novembre pour la Sainte-Catherine. Lors de cette dernière soirée, outre Olivier Guimond fils et l'orchestre de Laliberté, on y applaudit le chanteur Luc Bertrand, la chanteuse Judy Starr, le jongleur Rudy Sass et la danseuse June Lavall. On mesure l'intérêt du public en expérimentant, lors d'événements spéciaux, la formule

35 Compte tenu des divers numéros que présente un spectacle de variétés, il faudrait éviter d'associer ces références télévisuelles à la seule chaine française de RadioCanada. Non seulement le Ed Sullivan Show peut être évoqué, mais il en sera de même, par exemple, de l'émission Pick the Stars de la chaîne anglaise de Radio-Canada.

36 Le Clairon devient Le Clairon maskoutain à compter de mai 1954.

37 Le Clairon, 5 mars 1954, p. 6.

38 Le Clairon, 29 janvier 1954, p. 7. 


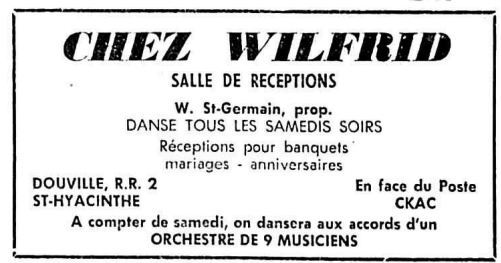

DANSE avec ORCHESTRE tous les Vendredis et Samedis soirs

au

GISAND HOTEL Inc.

A. Gaudette, prés. G. Gaudette, sec.-trés. 1395, GIROUARD TEL. 4.6464 ST-HYACINTHE

\section{DANSE tous les samedis soirs}

TOUS LES SAMEDIS SOIRS, à 8 hres 30

Jack Dulude et son orchestre

Pour Réception de noces, Banquet, Anniversaire

voyez Henri Mtiron, prop.

\section{Buliet Chez Menri Enr.}

- SPECIAL a LA MAISON -

Attention - 2 salles à votre disposition

2250, rUe STE-ANNE BOURG-JOLI TEL. 4.5803 Rés.: 1180 , rue Bourdages TEL. 4.8018 St-Hyacintho

\section{IHOTEL DTTAWA Enr.}

Jean et Roger Lefebvre, props.

LE RENDEZ-VOUS DES VOYAGEURS ET DES TOURISTES VOUS Y TROUVEZ:

Chambres avec Télévision, bain ef douche Sallets de Réceptions el de Banquets Salles d'échantillons pour voyageurs

1495, ST-ANTOINE TEL. 4.5351 ST:HYACINTH

Foyer Coquetel Dupont REPAS TOUS LES DIMANCHES SOIRS de 5 h. a $9 \mathrm{~h}$. Spécialité STEAKS RED BRAND

Commençant le 7 novembrt DANSE TOUS LES SOIRS

1185, LAFRAMBOISE TEL $4-9065$ STHYACINTH

Figure 3 - Une page publicitaire du Clairon maskoutain, le 19 octobre 1954, p. 2.

du cabaret, que l'on verra plus régulièrement à compter de 1957. Si l'on en juge par les soirées organisées à l'Hôtel Ottawa, qui serviront de modèle dans l'examen des soirées de variétés, les cabarets maskoutains connaîtront alors leurs meilleures années.

\section{Le cabaret de l'Hôtel Ottawa}

Le succès de la troupe de Jean Grimaldi, venue à quatre reprises au Paris entre décembre 1956 et janvier 1957, incite-t-il l'Hôtel Ottawa à offrir, à compter de février 1957, ses propres spectacles? Peut-être. Chose certaine, l'hôtel en organise chaque samedi soir à compter de cette date pendant plus de dix ans, ce qui nous porte à croire que le public a été nombreux et réceptif. Même si l'hôtel de la rue Saint- 
Antoine n'est pas le seul à présenter des spectacles, il permet d'en cerner l'évolution.

Au programme du samedi 2 février $1957^{39}$, deux représentations : l'une à vingt-deux heures et l'autre à minuit et demi, avec comme animatrice Yolande Gagnon (M.C.), accompagnée du chanteur André Roc, « du Café Mocambo de Montréal » (et frère de Michel Louvain), de la danseuse Rosita Salvador, du comédien Ray Dallaire et, bien sûr, de l'orchestre maison. Un an plus tard, on modifie les heures de chaque spectacle : vingt et une heures quarante-cinq pour le premier et vingt-trois heures trente pour l'autre. Cela renforce notre impression que la conception du spectacle de février 1957 était à l'essai.

Si cette soirée du 2 février demeure moins ambitieuse que celle animée par les 16 artistes de la troupe de Jean Grimaldi le même soir, il n'en demeure pas moins que ces soirées de cabaret servent de tremplin pour de jeunes vedettes comme, par exemple, Rosita Salvador, qui aura une belle carrière dans les années 1960 et 1970.

\section{La conception des encadrés publicitaires}

Avant de poursuivre, ouvrons une parenthèse sur ces encadrés publicitaires, source première de la présente recherche. Une comparaison entre ceux du Courrier de Saint-Hyacinthe et ceux du Clairon maskoutain livre de précieuses informations. L'examen des publicités de trois spectacles présentés en 1958 à l'Hôtel Ottawa permet d'en comprendre la conception.

Le spectacle du 12 avril met en vedette le chanteur Yvan Daniel. Si le texte est identique d'un journal à l'autre parce qu'il a, de toute évidence, été fourni par l'hôtel, on constate en revanche une grande liberté dans la disposition et même dans le choix de la photographie. En effet, la photographie du chanteur diffère d'un journal à l'autre (et est de mauvaise qualité dans les deux cas), laissant supposer qu'elles proviennent vraisemblablement de la photothèque de chaque hebdomadaire.

39 Le Clairon maskoutain, le 31 janvier 1957, p. 15. 


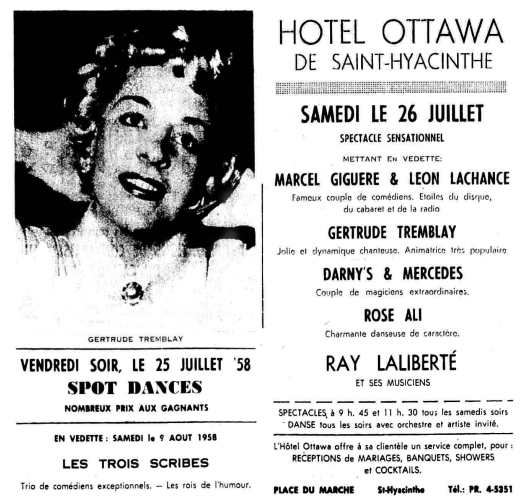

A L'HÖTEL OTTAWA DE SAINT-HYACINTHE

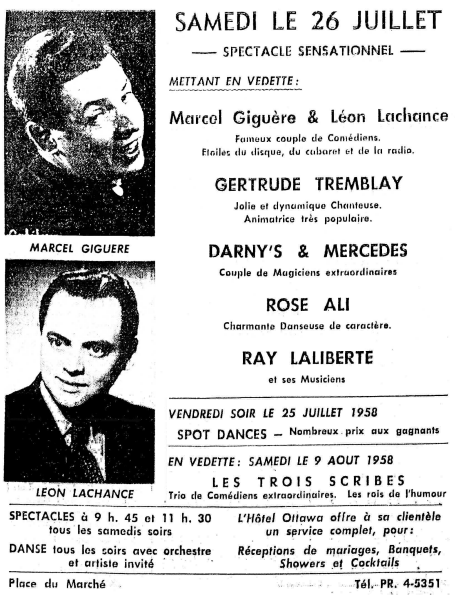

Figure 4 - Les encadrés publicitaires du spectacle du 26 juillet 1958. Sources : Le Clairon maskoutain, 24 juillet 1958, p. 3 ; Le Courrier de Saint-Hyacinthe, 24 juillet 1958, p. 3.

Prenons l'exemple du spectacle du 17 mai pour valider ces premières observations. Cette fois, les deux journaux insèrent la même photographie d'Olivier Guimond fils, mais cadrée bien différemment, celle du Courrier le montrant dans un plan plus rapproché. Les concepteurs de l'espace publicitaire des deux journaux ont assurément beaucoup de latitude, malgré les contraintes inhérentes à leur mise en pages respective, celle du Clairon maskoutain affichant une mise en pages moins allongée.

Prenons un dernier exemple, celui du 26 juillet (voir la figure 4). Il semble bien, en effet, que le choix de la photographie de l'artiste en vedette relève de chaque journal plutôt que du client, puisque, à neuf reprises en 1958, les deux hebdomadaires optent pour des artistes 
différents. Force est de constater que les journaux de cette époque sont de véritables ateliers publicitaires ${ }^{40}$.

\section{Une brève description des spectacles de variétés de 1958}

En 1958, le spectacle de cabaret de l'Hôtel Ottawa comprend généralement quatre volets, sans compter la partie réservée à l'orchestre. Chacun des volets se compose de quelques numéros dont l'un est assumé par le maître de cérémonie, habituellement une femme. Quelquefois, la tâche est confiée à un homme, comme le 5 avril lorsque le jeune Michel Louvain vient animer et chanter pour la première fois. Chaque semaine, on change de maitre de cérémonie, mais il arrive que celui-ci revienne une semaine ou deux de plus. Toujours au poste, l'orchestre de Ray Laliberté accompagne plusieurs des artistes invités et incite des couples à danser. Une danseuse, présentée comme jolie, charmante ou ayant du caractère, se charge du deuxième volet du spectacle.

Les deux autres parties comportent une combinaison de trois types, soit de la comédie, du chant et des numéros d'artistes divers, tels un acrobate, un magicien, un jongleur, un pantomime, un clown, un hypnotiseur, un marionnettiste, etc. Le volet comique peut être assumé par un fantaisiste comme Jean Rafa qui, cette année-là, vient même amuser la foule à deux reprises ${ }^{41}$. Quant aux chanteurs et aux chanteuses, des vedettes populaires y prennent l'affiche, comme Alys Robi (le 14 juin), Muriel Millard (4 novembre) et Rina Ketty (19 avril et 2 août) ainsi que de jeunes artistes prometteurs, comme un Pierre Sénécal (29 mars) ou un Marc Gélinas (16 août).

Si ces spectacles empruntent en tout point la formule du cabaret, ils se différencient néanmoins de ceux qui ont lieu dans une salle de

${ }^{40}$ Ce sujet, brièvement esquissé, mériterait une étude plus poussée car l'intervention des journaux varie sans doute selon les clients. Si l'on en juge par les encadrés des spectacles de Grimaldi, ces derniers comprennent beaucoup plus d'éléments communs d'un journal à l'autre, ce qui suppose l'envoi aux hebdomadaires non seulement d'un communiqué, mais aussi d'un canevas général de disposition.

41 Soit le 7 juillet et le 4 novembre. 
cinéma, non seulement par la vente d'alcool et par son public beaucoup plus restreint, mais aussi par l'offre de danse accompagnée d'un orchestre.

Toutefois, l'Hôtel Ottawa n'est pas le seul à proposer des variétés en 1958. La troupe de Jean Grimaldi, qui poursuit ses tournées, monte sur les planches du cinéma Corona à sept reprises ${ }^{42}$. Étant donné que les spectacles ont lieu la plupart du temps le samedi soir, temps fort de la semaine, on peut penser que Saint-Hyacinthe est devenue un arrêt incontournable. Cette année-là, presque tous les artistes de la troupe de Jean Grimaldi qui se produisent au Corona iront un jour ou l'autre à l'Hôtel Ottawa, soulignant ainsi le fait que les numéros demeurent interchangeables d'un lieu à l'autre ${ }^{43}$.

Autre constat, le volet comique se révèle moins dégarni qu'au cours de l'année précédente. Restons toutefois prudent, car, dans ce cas, seuls les principaux artistes sont nommés, ce qui n'est pas le cas des numéros de variétés. Impossible donc de savoir si, en 1957 comme en 1958, les spectacles offerts se terminent par une longue comédie, typique du théâtre burlesque, à laquelle Jean Grimaldi aurait pu participer.

L'encadré publicitaire du 25 octobre (voir la figure 5) permet de mieux en mesurer le volet comique. On peut certes compter sur La Poune pour faire rire le public, mais également sur certains chanteurs et musiciens et cela doit être souligné. À titre d'exemple, l'interprétation des Tune-Up Boys de la chanson très populaire « C'est en revenant de Rigaud ${ }^{44}$ " a sans aucun doute été fort distrayante. Et quand Les Jérolas participent au spectacle, comme c'est le cas le

42 Soit le jeudi 27 février, le samedi 10 et le mardi 20 mai, les samedis du 5 juillet, du 11 et du 25 octobre, et du 22 novembre. En moins de deux ans, la troupe de Grimaldi serait ainsi venue à 21 reprises à Saint-Hyacinthe, soit deux fois en décembre 1956, 12 fois en 1957 et 7 fois en 1958.

${ }^{43}$ Rose Ouellette et Juliette Petrie ont d'ailleurs raconté que leur passage du théâtre burlesque au cabaret s'est fait sans encombre; voir Petrie, Quand on revoit tout ça!, p. 191-192, et Ouellette, "La Poune », p. 73-77.

44 YouTube, [s. d.], [En ligne], [https://www.youtube.com/watch?v=t5Yh7O9FvIc] (5 septembre 2017). 


\section{* Cínéma CORONA Saint. Hyacintho * SAMEDI 25 OCTOBRE}

JEAN GRIMALDI prósente...

Un spectacle entièrement nouveau - du rire - chant - danse, etc. Les artistes les plus sensationnels

du Music-Hall ha Vedettes de la T.V. et des disques

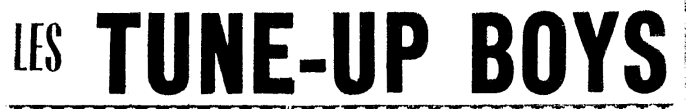

AU MEME PROGRAMME

La grande comédienine

LA POUNE

t

qui vous fera rire... aux larmes

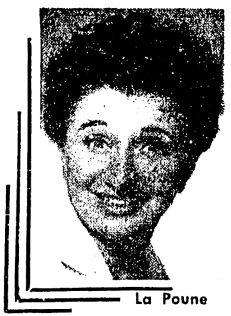

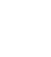

.
$\cdot$
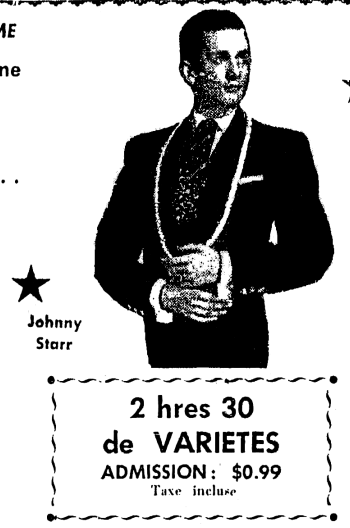
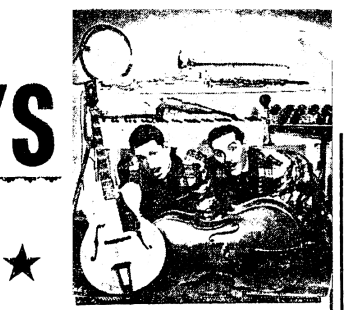

Yune-Up Boys

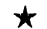

Le roi du tap-dance JOHNNY STARR

La charmante chanieuse MICKEY VAIL

L'accordéon magique ef CLAUDE EMOND

Du rire, du chant, de la danse et beaucoup de musique avec

SWING and SHAKE

Figure 5 - Le spectacle de la troupe de Jean Grimaldi du 25 octobre 1958, Le Courrier de Saint-Hyacinthe, 23 octobre 1958, p. 16.

10 mai et le 11 octobre, nul doute qu'ils amusent aussi beaucoup le public. Il n'est donc pas étonnant que certains chanteurs aient progressivement développé leur talent comique au fil de ces spectacles. Pensons à Fernand Gignac ${ }^{45}$ ou à Paolo Noël ${ }^{46}$, tous deux des habitués de ces soirées.

\section{Aperçu des tournées}

Il n'y a pas que le public maskoutain qui profite de ces spectacles. Ceux de Jean Grimaldi, par exemple, font partie d'une grande

45 Il participera à sept reprises au cabaret de l'Hôtel Ottawa entre 1958 et 1966.

46 Nous présentons plus loin ses visites à l'Hôtel Ottawa. 
tournée dont on peut saisir les modalités grâce à un dépouillement sommaire de quelques hebdomadaires régionaux de $1958^{47}$. Parce qu'ils ont été présentés dans les cinémas de cinq villes (en incluant Saint-Hyacinthe), deux spectacles ont été retenus : celui de La Poune, avec Yvan Daniel, et celui des Jérolas, avec Carmen Déziel. Or, ces tournées ne suivent pas un itinéraire précis puisque la première s'arrête d'abord à Saint-Hyacinthe, puis à Shawinigan et à Rimouski tandis que l'autre débute à Joliette, passe par Saint-Jean et se termine à Rimouski où, dans les deux cas, le spectacle prend l'affiche deux soirs consécutifs; la longue distance parcourue justifie sans doute l'amortissement des coûts sur deux soirées.

On aurait tort de croire cependant que l'éloignement de Rimouski en fasse un endroit idéal pour mettre un terme à la tournée; en effet, un autre spectacle de Jean Grimaldi, celui des Jérolas et de Jenny Rock, a d'abord été offert aux Rimouskois deux soirs de suite, avant d'être présenté aux Maskoutains deux mois plus tard. Une lettre conservée dans le fonds Jean-Grimaldi et portant sur une autre tournée va dans le même sens. Le 21 février 1958, il avait proposé, sans succès, au propriétaire du cinéma d'Amqui en Gaspésie de recevoir le spectacle le lendemain d'une représentation rimouskoise ${ }^{48}$. En fait, on serait porté à croire que les disponibilités changeantes des salles de cinéma et les estimations de profits chamboulent peut-être en permanence les circuits empruntés.

Le programme change-t-il d'une ville à l'autre? L'examen de trois encadrés d'un même spectacle au début de l'étée ${ }^{49}$ montre qu'il

${ }^{47}$ Ces journaux sont L'Étoile du Nord de Joliette, L'Écho du Saint-Maurice de Shawinigan, Le Progrès du Golfe de Rimouski et Le Canada Français de SaintJean-sur-Richelieu.

48 Lettre de Jean Grimaldi, 21 février 1958, Bibliothèque et Archives nationales du Québec, fonds Jean-Grimaldi, MSS433, 53, SS1, D11. Les éventuelles recettes de la soirée auraient été partagées entre Grimaldi et le propriétaire du cinéma dans une proportion de 60-40, mais la lettre ne précise pas qui aurait touché la plus grosse part.

49 L'un des encadrés est de l'Hôtel Royal de Sainte-Julienne et est paru dans L'Étoile du Nord, 25 juin 1958, p. 6; l'autre, du cinéma Impérial de Saint-Jean-sur-Richelieu, 
peut évoluer grandement ${ }^{50}$ au point même de s'accommoder à une reprise d'un hôtel plutôt que d'une salle de cinéma. Le spectacle de variétés, qui peut s'adapter aux circonstances et aux individus, reste une forme d'art de la scène extrêmement souple. Toutefois, seul le dépouillement complet de tous les journaux de l'Est canadien permettrait des jugements plus sûrs sur l'évolution des spectacles en tournée.

Il n'y a pas que les représentations de Jean Grimaldi qui prennent la route. Plusieurs vedettes à l'affiche au cabaret de l'Hôtel Ottawa en font autant. Mais le phénomène reste plus difficile à cerner en raison d'un manque d'information. Nous avons quand même pu dénicher les publicités des spectacles de Guilda ${ }^{51}$, de Rina Ketty ${ }^{52}$, de Marcel Giguère et de Léon Lachance ${ }^{53}$, d'Alys Robi ${ }^{54}$ et de Gilles Letarte $^{55}$. Or, comme ils partagent la scène avec des partenaires différents d'une ville à l'autre, ils semblent effectuer leur tournée en solitaire.

Revenons à Jean Grimaldi en abordant rapidement le début des années 1960. Un coup d'œil révèle la présence de quelques-uns de ses spectacles à Saint-Hyacinthe. Sans en faire une énumération détaillée, retenons toutefois qu'il propose une gamme de numéros qui comptent de moins en moins d'artistes. Le cinéma Corona, de son côté, a cessé

est paru dans Le Canada Français, 3 juillet 1958, p. 33. Le troisième encadré, du cinéma Corona, est publié dans Le Courrier de Saint-Hyacinthe, 3 juillet 1958, p. 7.

50 La comparaison des encadrés publiés dans Le Canada Français et dans Le Courrier de Saint-Hyacinthe confirme ici la grande latitude des journaux dans leur confection. Un coup d'œil sur l'encadré publié par Le Clairon maskoutain va dans le même sens.

51 À l'Hôtel Ottawa le 25 janvier, il est ensuite à l'Hôtel Monaco du fort Chambly les 7 et 8 février (Le Canada Français, 6 février 1958, p. 37).

52 Avant de se produire à l'Hôtel Ottawa le 2 août, elle avait chanté à l'Hôtel Royal les 11 et 12 juillet (L'Étoile du Nord, 9 juillet 1958, p. 2), puis au restaurant Kambo de Joliette le 20 décembre (L'Étoile du Nord, 17 décembre 1958, p. 2).

53 Après avoir participé au cabaret du 26 juillet à Saint-Hyacinthe, ils avaient présenté seuls leur numéro les 3 et 4 octobre à l'Hôtel Windsor de Marieville (Le Canada Français, 2 octobre 1958, p. 42).

54 À l'affiche de l'Hôtel Ottawa le 14 juin, elle est en vedette à l'Hôtel Windsor de Marieville le 17 octobre (Le Canada Français, 16 octobre 1958, p. 2).

55 À Saint-Hyacinthe le 12 juillet, il se rend à Sainte-Julienne le 6 décembre (L'Étoile du Nord, 3 décembre 1958, p. 2). 
ses activités dès 1961, tandis que les deux autres cinémas maskoutains ne présentent, cette année-là, que trois spectacles de variétés. En 1962, ils sont complètement disparus des salles de cinéma.

\section{Les hauts et les bas du cabaret de l'Hôtel Ottawa}

Quand on examine la liste des participants au cabaret de l'Hôtel Ottawa entre 1957 et 1968, on reste frappé par le retour régulier de plusieurs artistes, sûrement bien appréciés du public.

Les artistes de l'humour de l'époque dominent assurément ce classement ${ }^{56}$. À peine trois chanteurs, Paolo Noël, Yvan Daniel et Michel Louvain, et un danseur à claquettes, Johnny Starr, y ont été aussi régulièrement invités. Un chanteur comme Paolo Noël est en outre bien capable de dérider la foule ${ }^{57}$. Et la seule fois où le spectacle sera joué trois soirs de suite, soit un jeudi, vendredi et samedi, c'est la nouvelle troupe d'Olivier Guimond, Pique-Atout, qui attire les foules à l'automne 1961. On verra également cette troupe à RadioCanada, nouvellement convertie aux variétés ${ }^{58}$.

L'examen des quelque 500 soirées-cabaret indique que la plupart des artistes y viennent deux fois l'an ${ }^{59}$, le temps d'effectuer l'équivalent

56 Aird ne s'est pas trompé en décrivant les artistes de l'humour comme des vedettes fort populaires des cabarets (Aird, «De Coutlée», p. 33).

57 Voir, à ce sujet, le témoignage de Juliette Petrie, dans Petrie, Quand on revoit tout ça!', p. 195-200.

58 Un Jacques Normand, par exemple, aura son émission radio-canadienne Les Couche-Tard à compter de 1960. Par ailleurs, le fait que ce soit lors du spectaclebénéfice organisé au profit des réalisateurs en grève de Radio-Canada en 1959 que plusieurs artisans radio-canadiens découvrent enfin le génie d'un Olivier Guimond sur scène n'est pas sans rappeler les préjugés qu’ils affichaient jusqu'alors à l'égard de ce théâtre populaire, préjugés qui s'estompent seulement au tournant des années 1960. Voir Ouellette, "La Poune», p. 105; Pierre Day, Paul Berval: vivre pour le rire, Montréal, Trait d'union, 2004, p. 136-137 et Petrie, Quand on revoit tout ça!, p. 202.

59 Dommage que nous ne connaissions pas le nom de l'agence ou des agences d'artistes qui organisaient et planifiaient ces spectacles. Au sujet de leur rôle, on pourra lire le témoignage de Denise Filiatrault, dans Filiatrault et Lorain, Quand tu es née pour un petit pain, p. 78-79. 
Nombre et dates de spectacles des principaux artistes à l'Hôtel Ottawa, 1957-1968

$\begin{array}{ll}\text { Nom de l'artiste } & \begin{array}{l}\text { Nombre et dates } \\ \text { de spectacles }\end{array} \\ \text { La Poune } & \mathbf{2 0} \\ & (3-5-58,20-6-59, \\ & 12-12-59,12- \\ & 11-60,15-4-61, \\ & 30-9-61,11-8-62, \\ & 20-10-62,16-2-63, \\ & 2-11-63,21-3-64, \\ & 23-5-64,19-3-66, \\ & 16-4-66,9-7-66, \\ & 29-20-66,29-4-67, \\ & 9-9-67,18-11-67, \\ 4-5-68)\end{array}$

Paolo Noël

Yvan Daniel

Claude Blanchard $\mathbf{1 4}$

18

(20-12-58, 23-5-59, $14-11-59,2-4-60$, $1-10-60,28-1-61$, $15-7-61,16-12-61$, $2-5-62,3-11-62$, 4-5-63, 19-10-63, 29-2-64, 19-9-64, $13-2-65,15-5-65$, 2-4-66, 12-11-66)

\section{5}

(19-10-57, 12-4-58, 7-11-59, 23-7-60, 13-1-62,14-7-62, 13-4-63, 6-7-63, 5-10-63, 28-3-64, 5-9-64, 9-1-65, 24-7-65, 9-4-66, 10-12-66)

(28-11-59, 3-9-60, 1-7-61, 1-9-62, 7-12-63, 8-2-64, 14-11-64, 21-8-65, 23-10-65, 2711-65, 12-3-66, 10-9-66, 1-4-67, 23-9-67)

\section{Nom de l'artiste Nombre et dates de spectacles}

Léo Rivest

\section{0}

(30-11-57, 16-5-58, 11-4-59,11-7-59, 4-6-60, 24-9-60, 4-3-61, 1-7-61, 6-1-62, 1-9-62, 7-12-63, 8-2-64, 14-11-64, 21-8-65, 23-10-65, 27$11-65,12-3-66$, $10-9-66,1-4-67$, 23-9-67)

Olivier Guimond 15

(16-5-58, 4-6-60, 4-3-61, 7-10-61, $30-11-61,31-3-62$, 7-7-62, 8-12-62, 9-2-63, 18-5-63, 12-10-63, 30-1163, 8-5-65, 20-11$65,8-4-67)$

Père Gédéon 15

(5-7-58, 25-3-61, 17-6-61, 23-9-61, 21-4-62, 11-11-62, 20-7-63, 2-5-64, 19-6-65, 13-11-65, 12-2-66, 16-7-66, 19-11-66, 30-9-67, 27-4-68)

Michel Louvain 13

(5-4-58, 20-8-60, 6-5-61, 19-8-61, 14-4-62, 18-8-62, 12-1-63, 27-7-63, 18-4-64, 25-9-65, 30-4-66, 1-10-66, 7-10-67) 


\begin{tabular}{|c|c|c|c|}
\hline Wildor & $\begin{array}{l}13 \\
(3-1-59,11-7-59, \\
24-9-60,24-2-62, \\
22-9-62,1-6-63 \\
14-12-63,25-7-64 \\
4-6-66,17-12-66 \\
28-1-67,30-12-67)\end{array}$ & Gilles Latulippe & $\begin{array}{l}12 \\
(23-3-63,29-6-63, \\
14-9-63,30-5-64, \\
10-10-64,27-3-65, \\
3-7-65,4-9-65, \\
26-3-66,23-7-66, \\
26-11-66,18-3-67)\end{array}$ \\
\hline Marcel Gamache & $\begin{array}{l}12 \\
(14-12-57,18-2-58, \\
31-5-58,4-7-59, \\
5-8-61,20-1-62, \\
18-8-62,2-2-63, \\
13-6-64,1-5-65, \\
13-8-66,18-2-67)\end{array}$ & Johnny Starr & $\begin{array}{l}11 \\
(8-6-57,25-1-58, \\
7-3-59,28-11-59, \\
3-9-60,17-6-61, \\
28-7-62,16-3-63 \\
17-8-63,2-11-63 \\
19-9-64)\end{array}$ \\
\hline
\end{tabular}

de deux tournées en les espaçant afin de se faire oublier ou de se laisser désirer. Seule La Poune s'y fait applaudir à quatre reprises en 1966 alors que le cabaret maskoutain de l'Hôtel Ottawa vit ses dernières heures.

Mais ce tableau ne tient pas compte des maîtres de cérémonie : une Pierret Beauchamp, présente à huit reprises uniquement à titre de chanteuse, agit également comme maître de cérémonie et chanteuse à 43 occasions $^{60}$. Sa présence garantit une certaine constance dans le spectacle, d'un samedi à l'autre.

$\mathrm{Si}$, dans la liste des autres artistes qui se sont produits à l'Hôtel Ottawa, on ne se surprend pas de noter la présence du célèbre duo des cabarets, Ti-Gus et Ti-Mousse ${ }^{61}$, venu à sept reprises, on peut s'étonner que Jean Duceppe y soit venu six fois au début des années 1960. C'est peut-être oublier que le théâtre ne fait pas vivre son homme et que Duceppe a également été un animateur radiophonique

${ }^{60}$ Une fréquence qui s'explique par le fait qu’à compter du début des années 1960, ce rôle est attribué à la même personne pendant plusieurs semaines consécutives. La palme de l'artiste venu le plus souvent revient à Roxanne Scott, qui anima le cabaret à 62 reprises entre 1964 et 1967.

${ }^{61}$ Pour de belles descriptions de l'atmosphère des cabarets, on lira avec intérêt Lavallée, Denyse Émond Ti-Mousse et, plus particulièrement, les chapitres 14 et 15. Voir aussi le témoignage de Rose Ouellette dans Ouellette, "La Poune», p. 77-78. 
très populaire, qu'il était président de l'Union des artistes et un comédien apprécié pour son rôle de Stan Labrie dans le téléroman Les Plouffe, diffusé jusqu'en $1959^{62}$.

Le nombre de numéros peut servir, par la suite, d'indicateur sur la façon dont s'est développé ce cabaret. En effet, à compter du printemps 1962, l'établissement ajoute un deuxième orchestre, systématiquement remplacé au bout de quelques mois. Mais cela ne dure guère, car dès le $1^{\text {er }}$ décembre 1962, on supprime une des deux représentations, une décision majeure, s'il en est. Jusqu'à l'automne 1965, la soirée-cabaret conserve un seul spectacle composé de quatre numéros et de deux orchestres, ce qui laisse plus de place à la musique et à la danse. Puis, à compter de cette date, après quelques essais, on élimine pour de bon un des orchestres et on abaisse à trois le nombre de numéros. Finalement, en juin 1968 (voir la figure 6), se tient le dernier cabaret de l'Hôtel Ottawa, qui avait lieu régulièrement depuis 1957.

\section{La disparition des spectacles de variétés à Saint-Hyacinthe}

Pourquoi ce déclin et cette disparition des spectacles en cabaret et sur les scènes de cinéma maskoutaines? La question est complexe et mérite, en fin de parcours, quelques réflexions. Vue de SaintHyacinthe, l'année 1962 marque un tournant car on n'y présente plus guère de spectacles dans les salles de cinéma et ils sont amputés de moitié au cabaret de l'Hôtel Ottawa. Peut-être s'agit-il du contrecoup de l'arrivée récente d'une nouvelle chaîne télévisuelle de langue française, Télé-Métropole, l'ancêtre de TVA, qui, en faisant une grande place aux artistes de variétés ${ }^{63}$, rend les cachets et les

${ }^{62}$ La série diffusée le mercredi soir est tellement populaire qu'à l'époque, raconte Jean Rafa, « la clientèle des clubs de nuit, ce soir-là, assiste au deuxième spectacle, laissant la salle à peu près vide à l'heure du premier " (Day, Jean Rafa, p. 197). Juliette Petrie tient également des propos similaires, voir Petrie, Quand on revoit tout ça!, p. 202.

63 Lavallée, Denyse Émond Ti-Mousse, p. 145. 


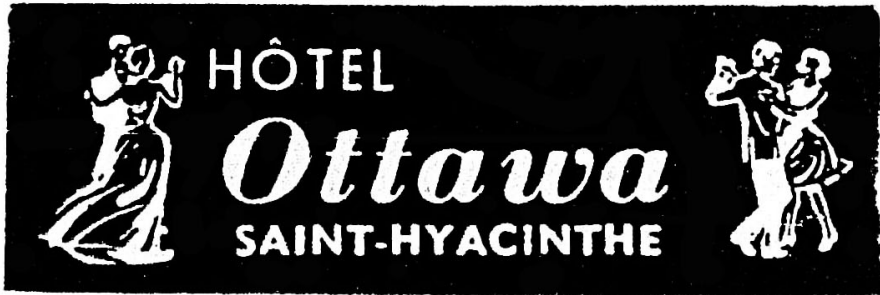

Place du Marché

Tól. : 774-5351

\title{
SAMEDI 29 JUIN 1968
}

SPECTACLE SENSATIONNEL

METTANT EN VEDETTE :

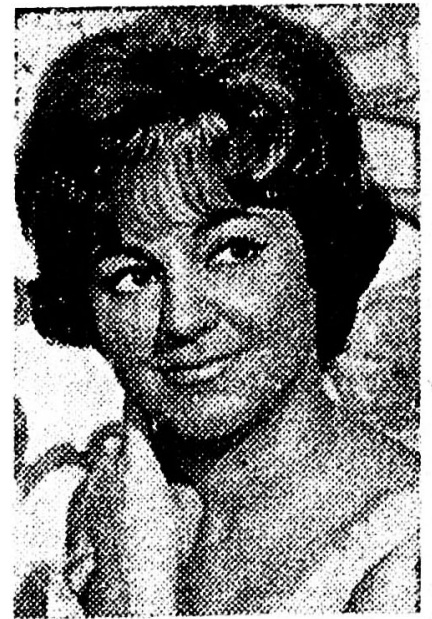

Gaby Laplante

\author{
Populaire chantense \\ de grande réputation
}

Ftoile de la radio, de la telricision. du disque et du raharet

(6) 0 o

\section{ROSELINE}

Charmante chantellop.

ef dynamigue animatrice

wow 6 or a

\section{LILI DIAMOND}

Sintillante dancense matique 4.

\section{ANDRE MORIN}

et ann enomble

Figure 6 - Le dernier cabaret de l'Hôtel Ottawa, Le Courrier de SaintHyacinthe, le 27 juin 1968, p. 3.

conditions de travail sur scène beaucoup moins attrayants ${ }^{64}$. Inaugurée le 19 février 1961, la chaîne a un effet majeur et va permettre, par exemple, à une Rose Ouellette de se produire enfin à la télévision ${ }^{65}$.

${ }^{64}$ Ibid., p. 113.

65 Ouellette, «La Poune», p. 106-107. 
Pour faire face à cette nouvelle concurrence ou encore pour réagir à la baisse de la clientèle, les propriétaires de l'Hôtel Ottawa repoussent d'abord l'heure des spectacles, à la fin d'avril 1961, à vingt-deux heures trente et à minuit et demi. Cette tentative sera vaine, si l'on en juge par l'élimination, un an et demi plus tard, d'un des deux spectacles, réduisant ainsi les cachets versés.

La popularité des boîtes à chansons, dans les années 1960, aurait contribué, selon Roger Chamberland, à la disparition des cabarets ${ }^{66}$. Il est vrai que les boîtes à chansons, qui se multiplient partout y compris à Saint-Hyacinthe, comme en font foi deux d'entre elles, Le Donjon et La Boîte à Rakakas, drainent une partie du public, dorénavant friand d'un genre nouveau. Parallèlement à ce développement, Saint-Hyacinthe assiste à la naissance de nombreux groupes locaux de musique yé-yé, tels les Aristocrates, les Houlops et les Sultans, pour n'en nommer que trois, au point d'être nommée la "Liverpool du Québec ${ }^{67}$ ».

À la lumière des témoignages de Denyse Émond et de La Poune, il faut relever, outre le développement de la télévision, des spectacles des chansonniers et des discothèques ${ }^{68}$, deux autres facteurs : les coûts prohibitifs que des imprésarios sans scrupule exigent des propriétaires de cabaret ${ }^{69}$ et le désir de l'artiste d'être seul sur scène ${ }^{70}$ et de rejoindre un public bien plus attentif quau cabaret ${ }^{71}$.

${ }^{66}$ Roger Chamberland, "De la chanson à la musique populaire ", dans Lemieux (dir.), Traité de la culture, p. 702.

${ }^{67}$ Voir Société d'histoire régionale de Saint-Hyacinthe, Saint-Hyacinthe, 17481998, p. 374-375.

${ }^{68}$ Lavallée, Denyse Émond Ti-Mousse, p. 112.

69 Ibid.; voir aussi Ouellette, "La Poune", p. 79.

${ }^{70}$ Lavallée, Denyse Émond Ti-Mousse, p. 112.

71 Voir, à ce sujet, le témoignage de Gilles Latulippe, "Lumière sur Gilles Latulippe », émission de télévision diffusée le 8 septembre 2015 au réseau ICI ARTV. De son côté, Denise Filiatrault avance un autre facteur pour expliquer le déclin des cabarets, soit une nouvelle législation provinciale forçant les hôteliers à servir un repas en accompagnement des spectacles, augmentant ainsi les coûts de production (Filiatrault et Lorain, Quand tu es née pour un petit pain, p. 136). 
Grâce à l'industrie du disque au Québec, mise en place à la fin des années 1950, beaucoup d'artistes font, en effet, la promotion de leurs microsillons, qui ont remplacé, au début des années 1960, les 78 tours $^{72}$. Au stade municipal de Saint-Hyacinthe et au théâtre Paris, pour la seule année 1968, des vedettes comme Hugues Aufray, Ginette Reno, Les Ménestrels, Lucien Hétu, Les Alexandrins, Jean Ferrat, Charles Aznavour, Les Cyniques, Adamo, Gilles Vigneault, Gilbert Bécaud et Vicky ont ainsi offert des concerts, qui ont attiré des foules nombreuses.

Doit-on s'étonner que Saint-Hyacinthe et l'Hôtel Ottawa n'aient jamais été mentionnés ni dans les témoignages publiés par les artisans de ces spectacles ni dans les documents relatifs aux tournées conservés dans le fonds Jean-Grimaldi? En fait, nous serions tenté de croire que c'est justement parce qu'elles étaient en tout point semblables à celles de bien d'autres villes que les soirées de variétés de SaintHyacinthe sont restées dans l'ombre jusqu'à maintenant.

Quelques mots s'imposent sur la nature des spectacles de variétés. En regard de ceux décrits tout au long de ces pages, ces spectacles n’ont jamais aussi bien mérité leur nom. Si leur présentation dans les cinémas permet d'ajouter des films selon la longueur des numéros, dans les hôtels, ils doivent composer avec la danse et la vente d'alcool au public. Comme l'antériorité des spectacles dans les salles de cinéma maskoutaines ne fait aucun doute, le cabaret a tardé à s'implanter à Saint-Hyacinthe alors que le modèle était très couru à Montréal. Leur histoire en région, si elle n'a pas laissé paraître de spécificités, a contribué à une meilleure compréhension de leur développement.

Faisons maintenant le point sur les répercussions du petit écran dans leur évolution. Un premier constat s'impose : Jean Grimaldi n'a pas fait la distinction entre l'arrivée de Radio-Canada en 1952 et

\footnotetext{
${ }^{72}$ Chamberland, " De la chanson à la musique populaire », p. 702.
} 
celle de Télé-Métropole en 1961. Non seulement neuf ans séparent ces deux événements, mais c'est seulement dix ans plus tard qu'il sera un des premiers à blâmer le petit écran pour l'abandon de ses spectacles et de ses tournées. En réalité, les preuves nous manquent encore pour avancer que les théâtres burlesques montréalais auraient grandement été affectés par l'arrivée du petit écran dès le lendemain de la première diffusion. Non seulement la télévision prend un certain temps à rejoindre le public, mais la situation maskoutaine semble indiquer que ces spectacles de divertissement dans les salles de cinéma connaissent encore un grand succès à la fin des années 1950 .

Grimaldi n'est pas le seul à avoir tenu des propos très vagues sur la télévision; Hébert en a fait autant en affirmant : "La télévision allait finalement achever le burlesque, non pas seulement parce qu'elle retenait plus de spectateurs à la maison, mais aussi parce qu' elle tenta de réaliser une formule, un genre de spectacles qui, d'une part, nécessitait fondamentalement la présence du public et qui, d'autre part, était devenu très coûteux à produire ${ }^{73}$."

Selon nous, ce n'est pas tellement le lancement de Radio-Canada en 1952 qui aurait contribué au déclin de ces spectacles en région, mais davantage l'ouverture graduelle de la société d'État aux artistes de variétés et la mise en ondes de Télé-Métropole en 1961. Au cours des années 1950, on évoque certes la présence, ici et là, de vedettes vues à la télévision dans les publicités, mais c’est comme si le petit écran servait seulement à mousser la vente de talents artistiques. Au début des années 1960, la télévision paraît avoir beaucoup plus d'influence, à Saint-Hyacinthe du moins. En 1962, le cabaret de l'Hôtel Ottawa amorce un net déclin tandis que les salles de cinéma maskoutaines cessent d'accueillir des variétés. À vrai dire, c'est au petit écran que ces spectacles vont fleurir. Commentant son entrée à Télé-Métropole, Rose Ouellette déclare : «On me vit alors dans presque toutes les émissions. Dix sur dix, Réal Giguère illimité, Bonne

73 Hébert, Le burlesque au Québec, p. 84-85. 
soirée, Madame est servie, Claude Blanchard, Vaudeville ${ }^{74}$. " Arrêtonsnous là puisque que d'autres recherches sur le contenu télévisuel et, notamment, sur l'influence de la télévision en couleur appelleraient des conclusions plus sûres.

École de formation pour les jeunes chanteurs et chanteuses, mais aussi divertissement de masse, le spectacle de variétés dans les cinémas et dans les hôtels a été avant tout un espace de comédie pour le grand public et l'expression d'une culture populaire dont les hebdomadaires, véritables lieux de production publicitaire, ont pu révéler l'existence. Le volet régional de l'histoire des variétés devra dorénavant être pris en compte, car vers la fin des années 1950, Saint-Hyacinthe, à l'instar sans doute d'autres villes, prend le relais de Montréal et assure un gagne-pain à de nombreux artistes pour encore quelques années. Presque au même moment où le dernier cabaret de l'Hôtel Ottawa se tient, les soirées de variétés réapparaissent en grande pompe à Montréal avec l'ouverture du théâtre du même nom en 1967. Un habitué de l'Hôtel Ottawa, Gilles Latulippe, aidé de son ami et mentor Jean Grimaldi ${ }^{75}$, en fera un succès grâce, entre autres, à la fréquentation assidue d'un public régional venu en autobus des quatre coins de la province $^{76}$ !

\footnotetext{
74 Ouellette, «La Poune», p. 107.

75 "Jean Grimaldi, le "papa des artistes" ", p. 24-25.

76 "Lumière sur Gilles Latulippe ».
} 